\section{Three deadly trypanosomatids decoded}

Genome sequences of three important parasites have been published, revealing some peculiar aspects of trypanosomatid biology that provide important clues for drug development. Information contained in the genomes of Trypanosoma cruzi (the agent of Chagas disease), Trypanosoma brucei (the agent of sleeping sickness) and Leishmania major (the agent of leishmaniasis) is revealed after some 100 years of research aimed at preventing and treating these diseases, which are widespread in the Southern Hemisphere.

Three genome papers, accompanied by further analyses papers - most notably, the comparative genomic analysis of El-Sayed and Myler et al. - are the result of an international collaborative effort. They are somewhat unusual: although each paper focuses on the genome of a particular protozoan, it also presents a cross-species comparison of selected aspects of trypanosomatid biology. The paper on T. cruzi discusses DNA replication and repair machinery, as well as retrotransposon content; the paper on T. brucei compares metabolic pathways and aspects of cell biology; and the paper on T. major describes unusual aspects of gene expression in these organisms.

The haploid genomes of these three parasites range from 25 to $55 \mathrm{Mb}$. They share a core of $\sim 6,200$ conserved genes and their genomes show a high degree of synteny. Strikingly, most genes are arranged in long directional clusters, which are most probably transcribed polycistronically and subsequently processed into separate mRNAs. Although the three genomes seem to encode few transcriptional regulators, they encode an uncharacteristically high number of proteins with RNA-binding motifs. These observations support previous suggestions that trypanosome gene expression is mainly regulated post-transcriptionally. The lack of sophisticated transcriptional control has another interesting implication - increased expression levels are achieved through gene duplication or amplification.

Aspects of DNA replication and repair are curiously different in trypanosomatids from the rest of the eukaryotes. Genes that are implicated in response to oxidative stress are missing from these genomes, as are those that encode the non-homologous end-joining machinery. In addition, the replication-initiation machinery is more reminiscent of that found in the Archaea than the eukaryote version.

Although related, each of these parasites has evolved a different strategy for parasitic survival. For example, the switching of variant surface glycoprotein (VSG) expression in T. brucei is a textbook mechanism for evading the host's immune response. Although much is known about VSGs already, genome sequencing revealed that most of them form subtelomeric arrays and are defective - only 7\% of analysed VSGs were functional. Arrays of defective VSGs could serve as an important reservoir of variation.

The quirks and peculiarities of trypanosomatid biology are of great biological and evolutionary interest. But importantly, they also have clear practical implications. Trypanosome-specific protein kinases, bacteria-like mitochondrial DNA polymerases and specific surface-protein modifications can all be explored as potential drug targets. We can now look forward to some effective treatments and perhaps the prevention of three important diseases, and so our luck might be changing in what until now was a losing battle.

Magdalena Skipper

69) References and links

ORIGINAL RESEARCH PAPERS El-Sayed, N. M. \& Myler, P. J. et al. The genome sequence of

Trypanosoma cruzi, etiologic agent of Chagas disease. Science 309, 409-415 (2005) |Berriman, M. et al. The genome of the African trypanosome Trypanosoma brucei. Science 309, 416-422 (2005) |

Ivens, A. C. et al. The genome of the kinetoplastid parasite, Leishmania major. Science 309, 436-442 (2005) | El-Sayed, N. M. \& Myler, P. J. et al. Comparative genomics of trypansomatid parasitic protozoa. Science 309, 404-409 (2005)

\section{IN BRIEF}

\author{
TECHNOLOGY
}

Potent and persistent in vivo anti-HBV activity of chemically modified siRNAs.

Morrissey, D. V. et al. Nature Biotechnol. 24 July 2005 (doi:10.1038/nbt1122)

Small interfering RNA (siRNA) molecules could be used for therapeutic approaches if an efficient and safe way to deliver them to the organism could be developed. Morrisey and colleagues have now developed such a delivery method by incorporating siRNAs into lipid-like particles. These encapsulated siRNAs are very efficient at inhibiting hepatitis B virus replication in mice, and work at low and clinically relevant doses.

\section{PLANT EVOLUTION}

\section{Evolution through genetically controlled allometry} space.

Langlade, N. B. et al. Proc. Natl Acad. Sci. USA 102, 10221-10226 (2005)

Morphological differences between species involve modifications in shape and size, but the genetic basis of these changes is poorly understood. The authors have applied numerical shape and size analysis to leaves in Antirrhinum species and showed that most of the variation can be represented in a three-dimensional allometric space, and involves at least 15 QTLs. This representation provides a quantitative framework for relating genetic variation to evolutionary changes in shape and size.

\section{PLANT GENETICS}

The pattern of polymorphism in Arabidopsis thaliana.

Nordborg, M. et al. PLoS Biol. 3, e196 (2005)

This study is a systematic survey of genomic DNA sequence polymorphisms in Arabidopsis thaliana. The authors resequenced 876 short fragments in samples from stock centres and natural populations and showed that the level of polymorphism is highly variable between genomic regions: it is negatively correlated with gene density and positively correlated with segmental duplications. They also showed that linkage disequilibrium is unusually extensive. These data will help to identify the functional polymorphisms that underlie phenotypic variation in A. thaliana.

\section{GENE NETWORKS}

\section{Rewiring of the yeast transcriptional network through} the evolution of motif usage.

Ihmels, J. et al. Science 309, 938-940 (2005)

The authors studied the evolution of gene expression by investigating differences in transcriptional regulation in two related yeast species. Loss of a cis-regulatory element from many genes was found to underlie changes in the regulation of gene expression that allowed the capacity for anaerobic growth to evolve in Saccharomyces cereviseae, but not in Candida albicans. This seems to have occurred after whole-genome duplication, indicating that such events allow rewiring of regulatory networks, as well as specialization of gene functions. 